\title{
Pregnancy in the time of COVID-19: A retrospective review comparing cases of in-utero death and placental abruption in 2020 to those in 2019
}

\author{
Charlotte Bishop ${ }^{1}$, Yasmin Mulji ${ }^{1}$, and Pippa Letchworth ${ }^{1}$ \\ ${ }^{1}$ Imperial College Healthcare NHS Trust
}

November 9, 2020

\begin{abstract}
Coronavirus disease 2019 (COVID-19) has a microembolic pathogenesis. We reviewed the incidence of microembolic complications of pregnancy during the COVID-19 pandemic period compared to the pre-pandemic period, specifically in-utero death (IUD) and placental abruption. Design A retrospective case review. Setting Patients were identified from the Datix reporting system and anonymised data was collected from the electronic health records. Population or Sample Women suffering an IUD or placental abruption during the review periods. Methods A retrospective review of the frequency and clinical characteristics of cases of IUD and placental abruption between 1st Jan 2020 - 30th June 2020 was compared to those from the 1 st Jan 2019 - 30th June 2019. Main Outcome Measures To evaluate the frequency and clinical characteristics of women suffering IUD and placental abruption during the pandemic period in 2020, and compare them to the pre pandemic period in 2019. Results There was a increase in the rates of IUD from 2019 to 2020, 1.22/1000 births compared to 2.85/1000 births ( $\mathrm{p}=0.10$ ). There was no difference in the rates of placental abruption $(\mathrm{p}=0.03)$. Conclusions Our review has demonstrated a non-significant rise in the frequency of IUD in the pandemic period in 2020 compared to those in 2019. Additionally, there was a significantly lower lymphocyte count during the pandemic period- a marker known to be associated with COVID-19. This warrants further investigation to explore the link between COVID-19 and disorders of poor placental perfusion, particularly the rates of IUD.
\end{abstract}

Pregnancy in the time of COVID-19: A retrospective review comparing cases of in-utero death and placental abruption in 2020 to those in 2019

\section{Running title:}

Review of cases of in-utero death and placental abruption during COVID-19

Bishop $\mathrm{C}^{1}$, Mulji $\mathrm{Y}^{2}$, Letchworth $\mathrm{P}^{3}$

1. Obstetrics and gynaecology ST2, St Mary's Hospital, London

2. Obstetrics and gynaecology ST3, St Mary's Hospital, London

3. Obstetrics and gynaecology consultant, St Mary's Hospital, London

\section{Corresponding author:}

Charlotte Bishop. St Mary's Hospital, Praed St, London. ST2. C.bishop2@nhs.net

Abstract

Coronavirus disease 2019 (COVID-19) has a microembolic pathogenesis. We reviewed the incidence of microembolic complications of pregnancy during the COVID-19 pandemic period compared to the prepandemic period, specifically in-utero death (IUD) and placental abruption. 


\section{Design}

A retrospective case review.

\section{Setting}

Patients were identified from the Datix reporting system and anonymised data was collected from the electronic health records.

\section{Population or Sample}

Women suffering an IUD or placental abruption during the review periods.

\section{Methods}

A retrospective review of the frequency and clinical characteristics of cases of IUD and placental abruption between $1^{\text {st }}$ Jan $2020-30^{\text {th }}$ June 2020 was compared to those from the $1^{\text {st }}$ Jan $2019-30^{\text {th }}$ June 2019. Statistical significance for means was calculated using a 2 sided t-test and using a chi-squared test for frequency data.

\section{Main Outcome Measures}

To evaluate the frequency and clinical characteristics of women suffering IUD and placental abruption during the pandemic period in 2020, and compare them to the pre pandemic period in 2019.

\section{Results}

There was a non-significant increase in the rates of IUD from 2019 to 2020, 1.22/1000 births compared to $2.85 / 1000$ births $(\mathrm{p}=0.10)$. There was no difference in the rates of placental abruption $(\mathrm{p}=0.03)$. There was also a statistically significant reduction in the lymphocyte count in cases from 2020 compared to 2019.

\section{Conclusions}

Our review has demonstrated a non-significant rise in the frequency of IUD in the pandemic period in 2020 compared to those in 2019. Additionally, there was a significantly lower lymphocyte count during the pandemic period- a marker known to be associated with COVID-19. This acts as proof of concept and warrants ongoing investigation to explore the link between COVID-19 and disorders of poor placental perfusion, particularly the rates of IUD.

\section{Tweetable abstract}

This review demonstrates a non-significant rise in the frequency of in-utero death during the 2020 pandemic period compared to 2019 ( $\mathrm{p}=0.10)$.

\section{Keywords}

COVID-19, SARS-CoV-2, pregnancy, obstetrics, stillbirth, IUD, abruption, microemboli

\section{Introduction}

In December 2019, the novel coronavirus SARS-CoV-2 was identified in a series of pneumonia cases in Wuhan, China. COVID-19 is the name given to the disease caused by SARS-CoV-2. In the UK, the first wave of the COVID-19 pandemic occurred during the first half of 2020. Whilst many cases in the pregnant population are mild, or even asymptomatic' those with severe disease can become critically unwell and require ventilatory support . Although pregnant patients do not seem to suffer more severe disease than non-pregnant patients, those with COVID-19 are at an increased risk of serious obstetric complications such as miscarriage, preterm birth, pre-eclampsia, and peri-natal death. Histopathological studies of the placentas of patients with COVID-19 show an increase in maternal venous malperfusion (MVM), which is known to be associated with both poor fetal outcomes and maternal hypertensive disorders. There is therefore biological plausibility that the rates of both placental abruption and in-utero fetal death (IUD) will increase in the era of COVID 19 (regardless of whether or not the patients tested positive for SARS-CoV-2 infection given the possibility 
of asymptomatic infection and the complications of lack of testing in the early stages of the pandemic). The objective of this study is to evaluate the frequency and clinical characteristics of women suffering IUD and placental abruption in the pandemic period, and compare them to the pre pandemic period.

\section{Methods}

The Datix patient safety incident reporting system was used to identify all cases of placental abruption and in-utero death (IUD) at Imperial College Healthcare Trust (Queen Charlotte's and Chelsea Hospital and St Mary's Hospital). All cases of IUD and placental abruption that occurred between $1^{\text {st }}$ January - $30^{\text {th }}$ June 2020 (pandemic period) and $1^{\text {st }}$ January - 30 ${ }^{\text {th }}$ June 2019 (pre-pandemic period) were included. There were no exclusion criteria. Anonymised data was collected retrospectively from the electronic health records. Demographic and clinical data was recorded to be used for comparison with cases from 2019.

\section{Statistical Methods}

The two cohorts (2019 and 2020) have been described using means for continuous variable, and relative frequencies for categorical variables. Statistical significance for means was calculated using a 2 sided t-test, and with chi-squared test for frequency data. A $P$-value of $<0.05$ was considered statistically significant. Data was analysed using the "Data Analysis" package in Microsoft Excel.

Results

During the pandemic period ( $1^{\text {st }}$ January $2020-30^{\text {th }}$ June 2020$)$ there were 3866 deliveries, of these 15 cases of placental abruption and 11 cases of IUD were identified (including two patients who suffered both IUD and abruption). During the pre-pandemic period ( $1^{\text {st }}$ January $2019-30^{\text {th }}$ June 2019) there were 4111 deliveries, of these 15 cases of placental abruption and 5 cases of IUD.

In these patient cohorts none were tested for COVID -19 .

Of the cases of IUD in 2020, two were associated with abruption, two with fetal growth restriction, and one with severe pre-eclampsia (PET). Seven were not associated with any of the above.

Baseline characteristics are outlined in table 1.

Haematological and biochemical data are outlined in table 2 .

Discussion

\section{Main Findings}

There was a non-significant increase in the number of IUDs pandemic period compared to pre-pandemic period, 2.85/1000 births compared to $1.22 / 1000$ births $(\mathrm{p}=0.10)$.

None of the patients tested positive for SARS-CoV-2 either during their admission, or during their antenatal course. This was during the early stages of pandemic when there was lack of access and strict case criteria for testing.

There was no significant difference in the age, BMI, ethnic category, or parity between cohorts. There was no significant difference in the rates of multiple pregnancy, gestational diabetes, pregnancy induced hypertension, pre-eclampsia, small for gestational age, and in-utero growth restriction.

There was no significant difference in white cell count (WCC), neutrophils, neutrophil: lymphocyte ratio, platelets, alanine transaminase (ALT), C-reactive protein (CRP), activated partial thromboplastin time (APTT), or prothrombin time (PT). There was a significant reduction in the mean lymphocyte count in 2020 compared to 2019 ( $\mathrm{p}=0.03)$.

Pregnancy outcomes were similar between the two years, with an average gestation at delivery of $33+0 / 40$ in 2019 , and $35+2 / 40$ in 2020 . The was no significant difference in the mode of delivery, estimated blood loss, or mean birth weight. Pregnancy outcomes are outlined in Table 3. 


\section{Interpretation}

There have been limited studies looking at COVID-19 in the pregnant population.

When a new pathogen emerges we can look to previous pandemics for insight into the natural history and course of the disease. With previous coronavirus spectrum infections serious acute respiratory syndrome (SARS) and middle eastern respiratory syndrome (MERS) there was a higher incidence of preterm birth, pre-eclampsia and caesarean section than in the general population. In addition, the clinical outcomes were worse for pregnant women than non-pregnant women, with a high incidence of death, renal failure, and disseminated intravascular coagulopathy .

By contrast, SARS-CoV-2 does not appear to have worse clinical outcomes for pregnant women compared to non-pregnant women. In a recent case series of 158 patients who tested positive for SARS-CoV-2 in New York, 78\% had mild or asymptomatic disease. This is comparable to a study of 118 SARS-CoV-2 positive pregnant patients in Wuhan, 92\% of whom had mild disease. In both settings, the risk of severe disease was similar to that of the general population, and appears to be associated with medical co-morbidities and increasing age.

COVID-19 is known to be a pro-coagulopathic state, with significantly higher rates of venous thromboembolism than in the general population. One might therefore expect evidence of thrombotic complications in the placentas of obstetric patients infected with COVID-19. Indeed, histopathological examinations of these placentas have demonstrated that they are more likely to show features of maternal vascular malperfusion (MVM) . These findings may suggest that these patients are at a higher risk of complications of poor placental perfusion, and warrant increased surveillance. Placental abruption ranges from the subclinical to massive catastrophic cases, histological assessment of all placentas throughout the pandemic period may have revealed many more cases of sub clinical abruption, but resources limit the ability to perform this in an initial review. We may therefore be underestimating the frequency of abruptions, and thus the impact of the disease.

Khalil et al studied the incidence of stillbirth and preterm birth at St George's Hospital, London during the pandemic period $\left(1^{\text {st }}\right.$ Feb 2020 to $14^{\text {th }}$ June 2020$)$ and the pre-pandemic period $\left(1^{\text {st }}\right.$ Oct 2019 to $31^{\text {st }}$ Jan 2020). Investigators found a significantly higher incidence of stillbirth during the pandemic period compared to the pre-pandemic period . Similar to our review, none of their patients had tested positive to SARS$\mathrm{CoV}-2$. Although the results were significant, they could not infer direct causality between asymptomatic SARS-CoV-2 and stillbirth. Other possible explanations include a reluctance of patients to attend hospital for fetal concerns, or a change in the available obstetric services during the pandemic period.

Despite none of our patients testing positive for SARS-CoV-2 there was a significantly lower lymphocyte count in 2020 compared to 2019. Lymphopaenia is known to be a cardinal feature of COVID 19. In the obstetric population, lymphopaenia is associated with moderate or severe disease . If we could consider lymphopaenia a proxy for SARS-CoV-2 infection, our preliminary findings could suggest an association between COVID-19 and IUD.

Learning from previous epidemics, it is widely acknowledged that services for women and children are especially vulnerable to disruption. In the 2014 Ebola outbreak in west Africa there was a $44 \%$ reduction in inpatient services, with similar reductions in antenatal and postnatal services, and family planning availability - Analysis of Sierra Leone's Health Management Information System found that the Ebola epidemic lead indirectly to 3,600 additional maternal deaths, neonatal deaths, and stillbirths . Similarly, during the COVID-19 pandemic, we have seen restructuring of services to prioritise acute care of those affected. This was compounded by fear of contracting the disease, healthcare provider sickness, and disruption to the global medical supply chain. Because of this, estimates of indirect maternal deaths during the COVID-19 outbreak in low- and middle-income countries lie between 8.3-38.6\%. It is therefore likely that some aspect of the rise in IUD that we have seen is an indirect, rather than direct, result of COVID-19.

\section{Strengths and Limitations}


To our knowledge, this is the first review looking at the associations between COVID-19 and poor placental perfusion. Both cohorts were well matched for demographic and clinical confounding factors. Our analysis allowed for any seasonal variation in primary outcome.

One limitation of this study is that the sample size is small due to the relative rarity of the primary outcomes. Further analysis would include other disorders of poor placental perfusion (pre-eclampsia, in-utero growth restriction, and preterm birth as well as IUD and placental abruption) over the whole pandemic; however the pandemic is currently ongoing and we report preliminary findings based on the first 6 months. We chose our primary outcomes as potentially acute complications of microemboli/MVM. The other conditions of poor placental perfusion may be relatively chronic in their presentation, and thus the study period would need to be extended to capture these broader complications.

Government policy on (and the availability of) testing for SARS-CoV-2 changed during the time of data collection, and testing for asymptomatic patients was not in our hospital protocol at any point during the review dates. Analysis of patients that test positive for COVID 19 is ongoing via the UKOSS study. In any future reviews of poor placental perfusion, it would be useful to know whether the patients identified were indeed positive for the SARS-CoV-2 virus, and this would potentially allow us to infer causality.

Cases were identified using the hospital reporting system DATIX, which has inherent limitations in terms of identification and reporting. Finally, some of our patients had incomplete documentation. In particular, some of the known haematological and biochemical markers of COVID-19 were not routinely assessed in our asymptomatic populations. (D-dimers, troponin, brain natriuretic peptide, creatinine kinase, ferritin). We are therefore unable to use them as potential surrogate markers for SARS-CoV-2 infection.

\section{Conclusion}

The risk from COVID-19 in pregnancy is low. Our review has demonstrated a non-significant rise in the frequency of IUD in the first six months 2020 compared to those in 2019, in addition to a significantly lower lymphocyte count- a marker known to be associated with COVID-19. This acts as proof of concept and warrants ongoing investigation to explore the link between COVID-19 and disorders of poor placental perfusion, particularly the rates of IUD.

\section{Disclosure of interests}

None to declare.

\section{Funding}

No external funding.

\section{Ethical approval}

Not applicable.

\section{Contribution to authorship}

CB contributed to conception, planning, data collection, data analysis, and write-up of the review. YM contributed to conception, planning, data collection, and editing the review. PL contributed to conception, planning, and editing the review.

Table 1: Baseline Characteristics

\begin{tabular}{llllll}
\hline & & Jan- July 2019 & Jan- july 2020 & Jan- july 2020 & $\mathrm{P}=$ \\
\hline Primary outcomes & Abruption & 15 & 15 & 15 & 0.87 \\
& IUD & 5 & 5 & 11 & 0.10 \\
DEMOGRAPHICS & Maternal age & 31.53 & 31.53 & 32.96 & 0.40 \\
& BMI & 25.68 & 25.68 & 25.13 & 0.75 \\
& Parity & 1.53 & 1.53 & 1.25 & 0.61
\end{tabular}




\begin{tabular}{|c|c|c|c|c|c|}
\hline & & Jan- July 2019 & Jan- july 2020 & Jan- july 2020 & $\mathrm{P}=$ \\
\hline & BAME & $12(66 \%)$ & $12(66 \%)$ & $19(79 \%)$ & \\
\hline \multirow[t]{5}{*}{ OBSTETRUC HISTORY } & Previous PET & 1 & 1 & 1 & \\
\hline & Previous SGA & 2 & 2 & 1 & \\
\hline & Previous PTB & 1 & 1 & 2 & \\
\hline & Previous IUD & 2 & 2 & 1 & \\
\hline & Previous abruption & 1 & 1 & 1 & \\
\hline \multirow[t]{5}{*}{ PAST MEDICAL HISTORY } & Essential hypertension & 0 & 0 & 1 & \\
\hline & Pre-existing diabetes & 0 & 0 & 1 & \\
\hline & Other autoimmune conditions & 0 & 0 & 2 & \\
\hline & Clotting disorders & 0 & 0 & 0 & \\
\hline & Kidney disease & 0 & 0 & 1 & \\
\hline \multirow[t]{3}{*}{ DRUG HISTORY } & AN Aspirin & 3 & 3 & 4 & \\
\hline & AN Heparin & 1 & 1 & 1 & \\
\hline & AN Antihypertensives & 0 & 0 & 2 & \\
\hline \multirow[t]{3}{*}{ SOCIAL HISTORY } & Current smoker & 3 & 3 & 3 & \\
\hline & Alcohol abuse & 1 & 1 & 0 & \\
\hline & Drug abuse & 0 & 0 & 0 & \\
\hline \multirow[t]{6}{*}{ CURRENT PREGNANCY } & Multiple pregnancy & 2 & 2 & 1 & \\
\hline & Gestational diabetes & 2 & 2 & 1 & \\
\hline & Pregnancy induced hypertension & 2 & 2 & 1 & \\
\hline & Pre-eclampsia & 0 & 0 & 4 & \\
\hline & SGA & 0 & 0 & 2 & \\
\hline & IUGR & 2 & 2 & 4 & \\
\hline
\end{tabular}

Table 2: Biochemistry

\begin{tabular}{llllll}
\hline & Jan- July 2019 & Jan- July 2019 & Jan- july 2020 & $\mathrm{P}=$ \\
\hline BIOCHEMISTRY & WCC & 13.68 & 13.39 & 13.39 & 0.83 \\
& Neutrophils & 10.55 & 10.92 & 10.92 & 0.83 \\
& Lymphocytes & $\mathbf{2 . 1 2}$ & $\mathbf{1 . 5 9}$ & $\mathbf{1 . 5 9}$ & $\mathbf{0 . 0 3}$ \\
& Neutrophil: lymphocyte ratio & 5.74 & 7.21 & 7.21 & 0.05 \\
& Platelets & 237.95 & 204.79 & 204.79 & 0.13 \\
ALT & 95.13 & 13.33 & 13.33 & 0.30 \\
CRP & 25.81 & 23.93 & 23.93 & 0.91 \\
APTT & 31.92 & 29.20 & 29.20 & 0.15 \\
PT & 14.27 & 13.22 & 13.22 & 0.23 \\
\hline
\end{tabular}

Table 3: Pregnancy Outcomes

\begin{tabular}{llllll}
\hline & & & Jan- July 2019 & Jan- july 2020 & Jan- july 2020 \\
\hline PREGNANCY OUTCOMES & Gestation at delivery & $33+0$ & $33+0$ & $33+0$ & $35+2$ \\
& Preterm birth & 13 & 13 & 13 & 9 \\
& Induction of labour & 6 & 6 & 6 & 12 \\
& Spontaneous vaginal delivery & 3 & 3 & 3 & 10 \\
& Instrumental & 1 & 1 & 1 & 0 \\
& Caesarean section & 15 & 15 & 15 & 14 \\
& Estimated blood loss (mls) & 1081.58 & 1081.58 & 1081.58 & 975.00
\end{tabular}




\begin{tabular}{lllll}
\hline & & Jan- July 2019 & Jan- july 2020 & Jan- july 2020 \\
\hline Birth weight & 2237.89 & 2237.89 & 2237.89 & 2494.55 \\
\hline
\end{tabular}

